\title{
ESTRATEGIAS METODOLÓGICAS UTILIZADAS POR DOCENTES QUE ATIENDEN NIÑAS Y NIÑOS PREESCOLARES DE 2 A 4 AÑOS DE EDAD
}

\author{
Bertha Barrantes Alpizar ${ }^{1}$ \\ Éricka Corrales Torres ${ }^{2}$ \\ Viviana González Rodríguez ${ }^{3}$ \\ Zulay Pereira Pérez ${ }^{4}$ \\ Eilyn Rodríguez Fallas 5
}

Resumen

Se analizaron mediante 120 observaciones de aula, las estrategias que utilizan las docentes para atender niñas y niños de 2 a 4 años de edad. La principal conclusión indica que existe una brecha, entre lo que las educadoras dicen que hacen y lo que realmente muestran en su ejercicio profesional.

Palabras claves: educación, estrategias metodológicas, ejercicio profesional, observaciones de aula.

\section{Abstract}

There were analyzed by means of 120 observations of classroom; the strategies that the teachers use to attend kids of 2 to 4 years of age. The main conclusion; indicates that a breach exists, between which the educators say that they do and what really they show in its professional exercise.

Keywords: education, strategies, professional exercise.

\section{Licenciada en Educación Preescolar. \\ 2 Licenciada en Educación Preescolar. \\ 3 Licenciada en Educación Preescolar.}

4 Catedrática de la Universidad Nacional. Máster en Psicologia de la Universidad de Costa Rica. Ex Coordinadora e Investigadora del Programa de Investigación en Epistemología Genética y Educación del IIMEC, Universidad de Costa Rica. Ex Directora de la División de Educación Básica del CIDE-UNA. Actualmente se desempeña como docente e investigadora en el Centro de Investigación y Docencia en Educación. Tiene publicaciones en el campo de la psicologia educativa.

5 Licenciada en Educación Preescolar. 


\section{Introducción y justificación}

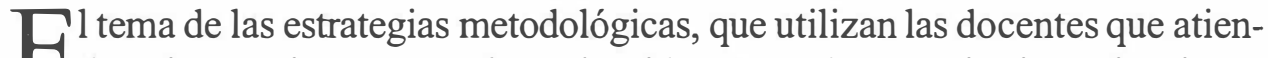
den niñas y niños preescolares, ha sido retomado en varias investigaciones costarricenses, pero la mayoría de ellas están referidas al nivel de preescolar, y no a quienes tienen entre 2 y 4 años de edad. Se han realizado estudios en diversos temas relacionados con infantes en edades comprendidas entre 2 y 4 años.

Algunos de esos estudios plantean que es evidente la necesidad de capacitar al personal de guarderías y centros que atienden niños pequeños ${ }^{6}$.

Por otra parte, hay estudios para esas edades, enfocados básicamente en aspectos curriculares y la incorporación de las diferentes áreas del desarrollo, que insisten en la necesidad de que dichos aspectos sean atendidos, especialmente, al trabajar con niños pequeños.

De igual manera, otras investigaciones enfatizan en las metodologías utilizadas con niñas y niños de 2 a 4 años de edad, señalando aspectos negativos en el campo metodológico, como la pasividad de las metodologías que se utilizan, lo poco innovadoras y la no pertinencia de estas, y la inadecuación de la atención brindada. Como resultado de los estudios, se fortalece la necesidad de profundizar en el abordaje de la atención de niñas y niños de 2 a 4 años de edad.

En Costa Rica, la mayoría de las investigaciones realizadas en torno a la educación preescolar se refieren a la etapa de transición, es decir, entre los 5 y 6 años de edad, pero hay pocas acerca de los niños menores de 4 años. Al respecto, Myers (2000) afirma que:

(...) la atención sigue estando centrada en niños de 4 a 6 años de edad, especialmente en el año anterior a la educación primaria. Cabe destacar que muy pocos niños están siendo atendidos en programas de atención y desarrollo para la primera infancia (p. 21).

\section{Consideraciones teóricas}

La situación que viven las familias de la sociedad costarricense en la actualidad es muy distinta a la de generaciones anteriores, donde la mayoría

6 González y Morales (1984); Escamilla y Fuentes (1985); Barrantes, González, Gutiėrrez y Villalta (1992). 
de los infantes menores de 6 años, crecían y se desarrollaban en un contexto únicamente familiar. Esta situación ha cambiado, pues el que una niña o un niño se quedara en su casa y fuera cuidado por su mamá, era algo común. Sin embargo, hoy es cada vez mayor el número de mujeres que se inserta en el mercado laboral, lo que ha convertido al Jardín Infantil en una instancia casi obligada y de apoyo para la dinámica familiar.

Tal como lo expresa Vila (2000), esta transformación se debe a varias razones. Una de ellas es la modificación de las condiciones de vida, que implica la salida constante de los padres y madres al trabajo, limitando así el tiempo compartido con hijas e hijos. Asimismo, existe cada vez mayor conciencia social acerca de la importancia de la educación infantil, para un desarrollo integral.

Realizar un análisis de las estrategias metodológicas que utilizan las docentes, con niños de 2 a 4 años de edad, es una aproximación para conocer la realidad de la educación inicial. Al respecto, Martínez (s.f.) comenta que:

(...) la inversión económica que se hace en programas de buena calidad y un currículo activo con niños menores en la edad preescolar, se traduce, a largo plazo en beneficios sociales, políticos y económicos para estos menores, su familia y la comunidad (p. 5).

Los primeros años de vida son claves para favorecer y consolidar el desarrollo de la persona en sus diferentes dimensiones: social, cognitiva, lingüística, motriz y de equilibrio personal. Trabajar en esta etapa educativa es un reto emocionante y, por lo tanto, no exento de dificultades y desafios. La intención de este trabajo es compartir inquietudes, comunicar experiencias y conocer las estrategias metodológicas para proporcionar atención integral a los infantes que se insertan en los centros educativos desde edades tempranas.

Las niñas y los niños, entre 2 y 4 años de edad, están vivenciando el período de mayor construcción de experiencias, de conocimientos y de desarrollo de la capacidad de emplearlos en la vida cotidiana. Por ello, es imprescindible brindar el ambiente y la metodología apropiados para atender sus necesidades, que les permita crecer, desarrollarse, madurar, aprender, construir conocimientos, afirmar las relaciones afectivas, socializarse. Estas funciones deberían ser propiciadas por la educación inicial en conjunto con la familia, la comunidad y las instituciones que los atiendan; no obstante, este tema ha sido poco abordado e investigado. 
El Ministerio de Educación Pública ha publicado el Programa de Estudios para el Ciclo Materno Infantil (Cerdas y Mata, 2000), que en teoría supone ser una guía para atender a los párvulos desde que nacen.

Asimismo, esta institución posee una guía didáctica para el desarrollo del aprendizaje de las y los infantes de 0 a 3 años de edad (Cerdas, 1995), que se fundamenta en una sistematización de autores, investigaciones, libros y ensayos acerca del trabajo educativo en este nivel. Sin embargo, en la realidad, se ha puesto en práctica sólo con niños mayores de cuatro años y medio, dejando de lado a los menores de 4 años. Es viable suponer que quizá tal población no está siendo atendida en concordancia con sus características, necesidades, intereses y niveles de desarrollo.

Por otro lado, existen otros documentos no formales relacionados con la atención de niños de 2 a 4 años de edad; no obstante, no unifican criterios ni reglamentaciones para atender a esa población. Entre estos documentos, se encuentran un Manual de normas de los establecimientos CEN-CINAI (Dirección de Centros de Educación, Nutrición y Desarrollo Infantil, 2002) y una Guia básica para el planeamiento didáctico (con niños y niñas de 2 a 6 años), según lo mencionan Aterhortúa, Navarro, Guerrero, Rodríguez, Padilla, Chacón; García y Brawon (2001).

También, existe un documento para la aprobación de los hogares comunitarios, que consiste en un listado de requisitos que debe cumplir la encargada del lugar para obtener su autorización de funcionamiento. Además, el Patronato Nacional de la Infancia provee a las delegadas de los albergues un material de apoyo, del Programa de la Mano para el Desarrollo de la Niñez en los Primeros 5 Años de Vida.

A pesar de la importancia de la atención de niñas y niños de 2 a 4 años de edad, se puede notar que son escasos los documentos que orientan a las docentes o encargadas para desarrollar la práctica educativa. Al respecto, Zúñiga (1995) comenta que en la atención a la primera infancia deben estar involucrados maestros especializados, pues influyen en el proceso de enseñanza y aprendizaje de los párvulos desde sus primeros años, ya que se ha comprobado que (...) "la educación es más efectiva cuando los maestros fundamentan sus prácticas en el conocimiento del desarrollo humano" (p. 113).

A pesar de esta recomendación, esta autora explica que en Costa Rica no todas las docentes de educación inicial poseen una formación o experiencia para trabajar con este tipo de población.

Unido a lo anterior, está la comprensión, diseño y apropiada implementación de estrategias metodológicas para niños de 2 a 4 años; en este sentido, 
Salas y Soto (1996) indican que las estrategias metodológicas son (...) "producto de una serie de decisiones referentes a las experiencias de aprendizaje más efectivas así como otra serie de aspectos vinculados con el proceso educativo en general" (p. 13). Queda clara la importancia de buscar el desarrollo integral del educando desde temprana edad.

Por otro lado, Bogantes y Jiménez (1996) afirman que las estrategias se pueden definir como (...) "todas aquellas técnicas, procedimientos y recursos didácticos que el docente considera oportuno utilizar, tomando en cuenta el nivel, intereses, habilidades, destrezas y vivencias de los alumnos" (p. 131), con el propósito de lograr un proceso de enseñanza-aprendizaje efectivo y exitoso.

Todavía más concretamente y enfatizando la labor del docente como promotor de estrategias de aprendizaje que favorezcan el desarrollo y aprendizaje de niños, está el planteamiento de De Zubiría y González (1995), quienes definen las estrategias metodológicas como la relación particular que se establece entre los tres factores principales: el saber, el alumno y el maestro; indican que (...) "según el papel que se les asigne, la manera como se relacionen entre sí y la importancia que cada uno de ellos cumpla dentro del proceso se hablará de diferentes estrategias metodológicas" (p. 16).

Lo planteado revela la necesidad que existe de capacitar y actualizar los conocimientos de las docentes para que la práctica educativa esté acorde con las edades y niveles de desarrollo de las niñas y los niños que atienden. Permite tener bases teóricas que fundamenten el trabajo de aula y la organización del programa de atención, según las características de la etapa evolutiva del estudiante, con el fin de diseñar y aplicar diversas estrategias metodológicas para atender a los pequeños, desde una perspectiva o enfoque determinado. Así también, busca y amplía la posibilidad de adecuar el currículo según la diversidad existente.

La educación infantil, entendida como el servicio público que responde a las necesidades educativas de la infancia y a las de sus familias, debe adoptar modalidades diversas, caracterizadas por la flexibilidad, la adecuación a los requerimientos reales de los niños y de sus familias. No obstante, las modalidades existentes en Costa Rica, que atienden a estos infantes, tienden a agruparse en dos extremos, es decir, algunas se inclinan a escolarizar a las niñas y los niños, basadas en planteamientos algo rígidos o, por el contrario, enfatizan su función en el cuidado asistencial sin que intervenga la parte educativa.

En definitiva, es indispensable destacar que, la educación institucionalizada está incorporando a los infantes desde edades muy tempranas. Por 
esta razón, se considera que las estrategias metodológicas usadas por las docentes, deberían estar acordes con las características, necesidades e intereses de esta población, con el propósito de promover un proceso de enseñanzaaprendizaje efectivo y significativo, que incentive el desarrollo integral infantil. En este sentido, los planteamientos teóricos señalan aspectos que han de ser considerados al trabajar con niñas y niños de 2 a 4 años. Entre esos aspectos, se mencionan los siguientes:

- La organización de los grupos debe promover una relación afectiva, estable, cálida y recíproca entre la niña, el niño y la docente. Estas características son fundamentales en las relaciones interpersonales, pues ahí se genera el proceso de interdependencia, construcción de confianza y seguridad.

- El ambiente físico es importante para el desarrollo de las estrategias metodológicas. En este factor, y de acuerdo con Peralta (1988), se deben considerar la infraestructura (mantenimiento, decoración, espacios con los que cuenta), la organización del aula (en espacios o rincones, filas, muebles) y el equipo con que cuenta. En este sentido, Cerdas y Mata (2000) precisan que (...) "es la organización de los 'espacios educativos' internos y externos lo que permite el movimiento, interacción, experimentación, descanso, alimentación, higiene, aseo personal en ambientes flexibles, cálidos, relajantes, atractivos y seguros" (p. 82).

Por lo tanto, se debe pensar en conformar un ambiente físico, social, mental y emocional, que brinde la posibilidad a la niña y al niño de actuar. La interacción entre adultos e infantes debe ser de respeto, natural, consistente y apropiada a su nivel de desarrollo, con el fin de brindar confianza, establecer límites claros, un ambiente libre de tensión y acogedor.

Cerdas y Mata (2000) plantean que los espacios de interacción exigen algunas condiciones específicas que contemplan la edad, las necesidades, las características y potencialidades de cada grupo, donde la docente debe proporcionar una supervisión constante. Deben permitir el desarrollo de experiencias lúdicas, creativas y constructivas.

Los horarios constituyen otro factor a considerar cuando se trabaja con niñas y niños pequeños; estos deben incluir experiencias alternadas: tranquilas y activas, espontáneas y estructuradas, colectivas, de pequeños grupos e 
individuales, en espacios exteriores e interiores, iniciadas por el adulto o los niños, y cotidianas e innovadoras. Se debe disponer de espacios de tiempo para experiencias de libre acción, experiencias estructuradas, intermedias y de rutina o cuidado diario.

En cuanto a la interacción con los materiales, Cerdas y Mata (2000) consideran que (...) "el material puede ser natural, de desecho o comercial orientado a favorecer la acción física, mental, social y emocional del sujeto, sin ser únicamente perceptual, sino generador de experiencias problematizadoras" (p. 92). Deben ser materiales que permitan la invención, el descubrimiento de relaciones, la creatividad y que estimulen conflictos cognoscitivos.

Como se nota, los aspectos que influyen en el desarrollo de experiencias de enseñanza y de aprendizaje son muy variados, los cuales han de ser considerados cuando existe un deseo en la institución educativa y en los docentes, por promover el desarrollo de la niña y del niño, así como su aprendizaje.

\section{Consideraciones metodológicas}

La investigación que dio origen a este artículo fue de tipo descriptivoexploratoria. Se trabajó con un grupo de 25 docentes de Educación Preescolar que estuvieran atendiendo o hubieran atendido en algún momento a la población de niñas y niños con edades entre los 2 y 4 años.

De igual manera, se llevaron a cabo 120 observaciones de aula, de aproximadamente dos horas de duración cada una de ellas. Dichas observaciones se hicieron en 12 grupos en total ( 6 guarderías privadas y 6 jardines infantiles). De los grupos observados en las guarderías, 3 se ubicaron en San José y los otros 3 en Heredia centro. En relación con los grupos de los jardines infantiles, 3 estaban localizados en San José y 3 en Heredia.

La recopilación de datos se hizo mediante un cuestionario dirigido a docentes en servicio, el cual fue aplicado por las investigadoras, así como la realización de 120 observaciones de aula utilizando una lista de cotejo para identificar y caracterizar las estrategias metodológicas que utilizaban las docentes al desarrollar la lección, en cuanto a cinco grandes categorías: espacios interiores, espacios exteriores, recursos materiales y mobiliario, planificación y factor humano. 


\section{Resultados}

Se destacan a continuación los resultados más relevantes en cuanto a las estrategias metodológicas utilizadas por docentes en el trabajo de aula con niñas y niños de 2 a 4 años de edad; se plantean no sólo los criterios que las docentes manifiestan utilizar en cuanto a estrategias, sino que también se contrastan con los datos recopilados a partir de las 120 observaciones de aula.

Los resultados nos dicen que de las 25 docentes de preescolar participantes, en la elección de las estrategias metodológicas para el desarrollo de la dinámica de aula, el $96 \%$ toma en cuenta como primer aspecto las necesidades de los pequeños; el $92 \%$, la edad de las niñas y los niños; el $88 \%$ retoma las características del grupo; el $84 \%$ de las educadoras hace referencia al conocimiento previo de sus estudiantes, así como el tiempo ${ }^{7}$. La metodología de la institución (plan anual, objetivos, lineamientos, orientación didáctica, entre otros); las características individuales de los infantes; los materiales y recursos, así como el espacio, fueron señalados por un $76 \%$ de las maestras.

Por otro lado, el $48 \%$ de las docentes considera la forma de trabajo para elegir las estrategias metodológicas y finalmente, el 12\% agrega otras categorías no contempladas en el cuestionario aplicado, tales como: misión que tiene planteada la institución e intereses que tienen los infantes.

Según sus respuestas, el principal criterio que consideran las docentes al seleccionar las estrategias metodológicas adecuadas son las necesidades de los pequeños. Es importante mencionar que los planteamientos teóricos actuales señalan que al planear han de considerarse las características de desarrollo de las niñas y los niños de 2 a 4 años de edad, el hecho de que los infantes exploran el medio, mediante su cuerpo, el lenguaje, actividades fisicas y mentales y que a esta edad, disfrutan de participar en actividades lúdicas como la imitación, el juego, que es la actividad que más les entretiene, mediante este le dan significado a la realidad, pues utilizan su creatividad e iniciativa para investigar el mundo que los rodea.

Por las razones anteriores, puede decirse que lo planteado por las docentes coincide ampliamente con el referente teórico. No obstante, en las observaciones realizadas en los grupos de guarderías y jardines infantiles,

7 Cabe señalar que las docentes mencionaron más de un aspecto, razón por la cual los porcentajes están en función de cada uno de esos aspectos. 
se denota que en su mayoría, las actividades planteadas se basan en la decisión de las educadoras, la realización de la rutina y poca participación de los chicos. En este sentido, se deduce que la dinámica de clase se enfatiza en el cumplimiento de un plan establecido, donde la realidad del aula contradice lo expresado por las educadoras mediante el cuestionario.

Ante esto, se establece que, aunque las maestras reflejan en sus respuestas que las necesidades de los pequeños son el primer criterio para seleccionar las estrategias que usan en el aula, la práctica demuestra que no consideran las necesidades individuales, la edad y los conocimientos previos, a pesar de que la participación activa de sus estudiantes es la mejor herramienta para desarrollar sus habilidades y conocimientos.

\section{Gráfico 1}

Apoyos que las docentes de preescolar dicen utilizar para la planificación y selección de las estrategias metodológicas

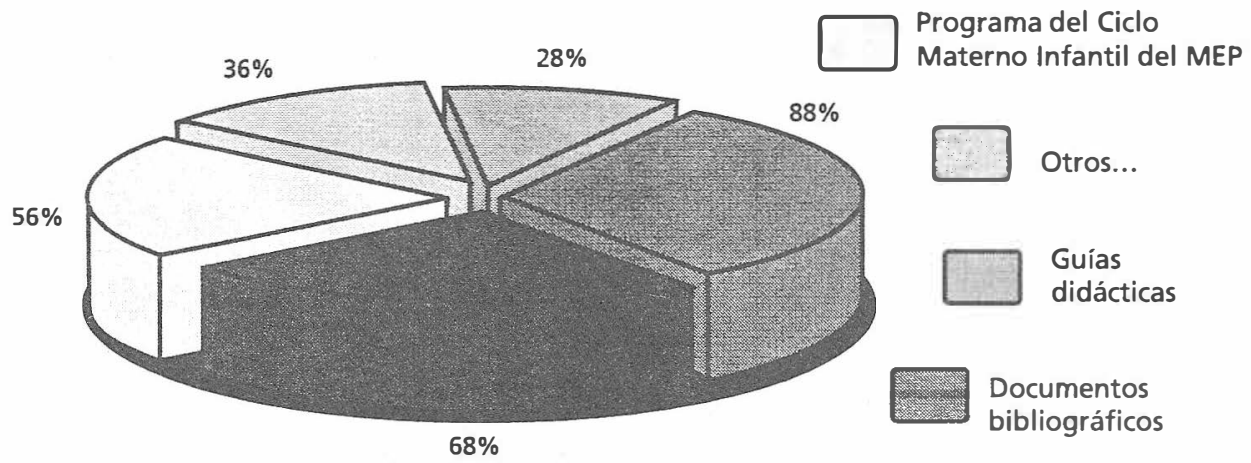

Plan anual de la institución

Cabe destacar que la totalidad de las docentes de preescolar participantes en el estudio manifiestan utilizar apoyos didácticos en la planificación y selección de las estrategias metodológicas que despliegan con el grupo de niñas y niños a su cargo; sin embargo, dichos apoyos varían según las necesidades e intereses de las educadoras. Desde esta perspectiva, se destaca que 22 maestras (88\%) emplean documentos bibliográficos (revistas, libros, intemet, enciclopedias, entre otros) como apoyo principal.

En segundo lugar, 17 entrevistadas (68\%) usan el plan anual de la institución; 14 educadoras (56\%) se apoyan en el Programa del Ciclo Materno 
Infantil del MEP; 8 docentes (36\%) acuden además a otros apoyos suplementarios, tales como: Programa de Transición de Inglés, crónicas de observación, material entregado por la Directora Académica, actividades propuestas por los niños, materiales elaborados extracurricularmente y los aportes de los padres de familia. Por último, 7 maestras $(28 \%)$ mencionan que recurren a las guías didácticas para seleccionar y planificar las estrategias metodológicas a implementar en sus aulas.

Se considera que la función de la institución educativa es ejercer una labor didáctica planificada, basada en principios pedagógicos actualizados y métodos acordes con las características y necesidades de las niñas y los niños. Cada institución privada tiene la libertad de escoger, según sus planteamientos, los fundamentos y fuentes que guiarán su práctica educativa.

Peralta (1988) agrega que, de acuerdo con el tipo de institución, se plantean fundamentos que responderán a la pregunta, ¿Cómo enseñar?, así como objetivos, contenidos, procedimientos y evaluaciones que permitirán el desarrollo de la práctica pedagógica y el cumplimiento de la visión y misión de cada entidad. El estudiante encierra las expectativas que se tienen en cuanto al tipo de persona que se desea formar y este se refleja en el tipo de apoyo didáctico que utilicen las docentes para orientar sus estrategias.

Se presentan a continuación, los datos recopilados mediante las observaciones de aula y que ejemplifican lo que realmente se lleva a cabo en el desarrollo de las lecciones en las instituciones educativas analizadas.

\section{Cuadro $\mathrm{N}^{0} 1$}

Análisis de los espacios exteriores en seis grupos de maternal y prekínder de jardines infantiles y seis grupos de guarderías, según frecuencia y porcentaje

\begin{tabular}{|c|c|c|c|c|}
\hline Criterios de análisis & \multicolumn{2}{|c|}{$\begin{array}{c}\text { Jardines infantiles } \\
\text { Sí }\end{array}$} & \multicolumn{2}{|c|}{$\begin{array}{l}\text { Guarderías } \\
\text { Sí }\end{array}$} \\
\hline Espaeios exteriores & F & $\%$ & F & $\%$ \\
\hline Zona de juegos & 6 & 100 & 6 & 100 \\
\hline Zonas verdes & 1 & 16.66 & 3 & 50 \\
\hline Zona techada & 3 & 50 & 4 & 66.66 \\
\hline Zona de jardín & - & - & 3 & 50 \\
\hline Zona de huerta & - & - & 1 & 16.66 \\
\hline $\begin{array}{l}\text { Zona de cuido de } \\
\text { animales }\end{array}$ & 1 & 16.66 & - & - \\
\hline
\end{tabular}

$\mathrm{n}=6$ grupos por tipo de institución. 
Con respecto a los espacios exteriores de los grupos observados, se destaca que los 12 grupos (100\%) tanto de los jardines infantiles (6 grupos) como de las guarderías (6 grupos), la parte exterior se organiza y distribuye en zona de juegos. Sólo un grupo (16.66\%) de los jardines tiene áreas verdes, mientras que 3 grupos (50\%) de las guarderías cuentan con estos espacios.

La organización de estos lugares coincide con el criterio del Ministerio de Educación Pública (citado por Cerdas y Mata, 2000), en relación con los espacios exteriores, ya que señala que es importante que existan zonas verdes y áreas de juegos, que permitan el movimiento, la interacción, experimentación, exploración, el fomento del desarrollo integral y óptimo de la niña o el niño en un ambiente seguro y agradable. Sin embargo, las zonas verdes se encuentran en un porcentaje bajo, lo que limita el contacto del infante con la naturaleza y algunas experiencias de aprendizaje mencionadas anteriormente.

\section{Cuadro $\mathrm{N}^{\circ} 2$}

Análisis acerca del tipo de recursos existentes en las áreas de seis grupos de matemal y prekínder de jardines infantiles y seis grupos de guarderías, según frecuencia y porcentaje

\begin{tabular}{lcccc}
\hline Criterios de análisis & Jardines infantiles & \multicolumn{2}{c}{ Guarderías } \\
\hline Recursos existentes & F & $\%$ & F & $\%$ \\
\hline De desecho & 3 & 50 & 2 & 33.33 \\
Comerciales & 6 & 100 & 6 & 100 \\
Naturales & - & - & 1 & 16.66 \\
Elaborados por las niñas(os) & 2 & 33.33 & 3 & 50 \\
Elaborados por la docente & 3 & 50 & 6 & 100 \\
\hline
\end{tabular}

$\mathrm{n}=6$ grupos por tipo de institución.

Es importante destacar que la mayoría de los recursos existentes en las aulas son comerciales. Los recursos elaborados por los niños fueron observados en 2 grupos (33.33\%) de los jardines infantiles y en 3 grupos $(50 \%)$ de las guarderías.

Los datos obtenidos reflejan que los materiales comerciales son los predominantes en ambas instituciones; sin embargo, también hay presencia de otros materiales como los de desecho, los naturales y los elaborados por la docente.

Se puede notar que un porcentaje minoritario de las áreas observadas, cuenta con materiales elaborados por los niños. A partir de los datos aportados, es válido recordar que es importante que el niño tenga una mayor participación 
en los procesos de enseñanza y aprendizaje porque los pequeños conocen el mundo principalmente mediante sus propias acciones.

\section{Cuadro $N^{\circ} 3$}

Análisis acerca de la planificación de actividades para las niñas y niños en seis grupos de maternal y prekínder de jardines infantiles y seis grupos de guarderías, según frecuencia y porcentaje

\begin{tabular}{llccc}
\hline Criterios de análisis & \multicolumn{2}{l}{$\begin{array}{l}\text { Jardines } \\
\text { infantiles }\end{array}$} & \multicolumn{2}{c}{ Guarderías } \\
\hline Planificación de actividades & $\mathrm{F}$ & $\%$ & $\mathrm{~F}$ & $\%$ \\
\hline $\begin{array}{l}\text { De rutina (horario establecido: formación } \\
\text { de hábitos, alimentación, saludos...) }\end{array}$ & 6 & 100 & 6 & 100 \\
Propuestas por la docente & 6 & 100 & 6 & 100 \\
Individuales & 2 & 33.33 & 4 & 66.66 \\
Subgrupales (trabajo en grupos pequeños) & 5 & 83.33 & 3 & 50 \\
Colectivas (trabajo con todo el grupo) & 6 & 100 & 6 & 100 \\
Otras (excursiones, fiestas, talleres) & 4 & 66.66 & 1 & 16.66 \\
\hline
\end{tabular}

$\mathrm{n}=6$ grupos por tipo de institución.

En el cuadro tres, se puede resaltar que se sigue una rutina. Las actividades en su totalidad son propuestas por la docente y son colectivas, es decir, la tendencia es trabajar con todos los párvulos que conforman el grupo y esto se da tanto en los jardines infantiles privados como en las guarderías.

Por otra parte, las actividades subgrupales ocupan un lugar importante, pues cinco de los seis grupos observados en jardines infantiles privados, poseen esta característica, lo que representa el $83.33 \%$. En las guarderías, tres de los seis grupos observados cumplen con este aspecto, lo que equivale a un $50 \%$.

Cabe destacar que en los jardines infantiles privados no se observó que las actividades fueran propuestas por la niña o el niño, ni espontáneas, mientras que en las guarderías, se observa que cuatro de los seis grupos, poseen la característica de que las actividades son propuestas por las y los estudiantes, lo que corresponde a $66.66 \%$, y cinco de los seis grupos practican actividades espontáneas, es decir, un $83.33 \%$. 
La planificación es un elemento que contempla el tipo de actividades que se utilizan para desarrollar la práctica educativa, en las cuales se debe considerar si son iniciadas por el adulto o por el niño, si son espontáneas, colectivas, desarrolladas en espacios interiores o exteriores y el tiempo en que se organizan. La planificación se desarrolla mediante prácticas físicas, mentales, sociales y emocionales, que permite la construcción de conocimientos, fortaleciendo el desarrollo de los pequeños.

Al respecto, Obando y Quesada (1995) señalan que las actividades que se realicen deben ser variadas, incluyendo el desarrollo de destrezas temporales espaciales, cotidianas, experiencias con agua y arena, la manipulación y exploración de instrumentos musicales, la observación y experimentación con materiales naturales, la expresión y exploración plástica, construcción, ejercitación muscular, simulación y dramatización.

El estudio permitió detallar que en las instituciones privadas, el grado de participación que desarrollan las niñas y los niños está guiado por las decisiones que toma la docente. El 100\% de los participantes cumple un papel pasivo, es decir, las y los estudiantes participan tomando como guía la acción de la docente. En este sentido, es ella quien dispone lo que se va a realizar y luego estimula la participación de los pequeños.

Al respecto, es importante traer a colación lo que mencionan Cerdas y Mata (2000), en cuanto a la acción de las niñas y los niños, pues se considera que son ellos los protagonistas del proceso de enseñanza-aprendizaje. Este pensamiento es apoyado por Hernández y Rodríguez (1998), al exponer que para estas edades es sumamente importante ofrecer las oportunidades para que desarrollen sus potencialidades y capacidades, pues durante este período los infantes construyen conocimientos acerca de sí mismos, de los demás y del mundo que les rodea.

No obstante, en las observaciones realizadas, se logró denotar que las docentes organizan la rutina de clase de una manera muy establecida, lo cual limita la participación creativa y espontánea de los párvulos. Esto no quiere decir que en las clases, los párvulos no participen, sino que su modo de intervención está muy ligado a lo que la educadora tiene preparado de antemano y no se deja lugar para las experiencias que surjan de imprevisto o por iniciativa del grupo.

En el caso de las guarderías, se observó que son las niñas y los niños quienes proponen otras opciones de trabajo o actividades, aparte de las que determina la educadora. De modo que si se incentiva la participación de los 
pequeños desde edades muy cortas, se estará favoreciendo la independencia, la relación con el medio en que se desenvuelven, así como el verdadero protagonismo en los procesos de enseñanza-aprendizaje.

Apoyando estas ideas, Peralta (1988) menciona que en la dinámica de clase es importante que se le otorgue a los pequeños un papel activo, lo que, (...) "va a significar que el niño tome muchas decisiones, incluso, algunas que habitualmente han sido consideradas como propias del educador" (p. 41).

Finalmente, se observó que en dos grupos de guarderías (33.33\%), la participación de la población está guiada por la acción de la docente. Esto da pie a pensar en la necesidad de invertir en mayores esfuerzos para promover la participación de las niñas y los niños en la toma de decisiones en las actividades de clase. Tal hecho implica planificar la metodología de clase, para lograr la unificación de las acciones de los colaboradores, con la finalidad de mejorar las relaciones interpersonales, la participación y la toma de decisiones de los pequeños y la docente.

La observación realizada acerca de la manera en cómo las docentes efectúan su labor y el modo en que se desempeñan permitió detallar, que las docentes de los seis grupos observados de jardines infantiles asumen un rol caracterizado por, ser ante todo, las personas que transmiten conocimientos a sus estudiantes, se enfocan en llevar a cabo actividades en las que prima el aspecto cognitivo, es decir, la realización de rutinas que les permite desarrollar conceptos o destrezas importantes para los alumnos, los padres y las madres. Se deja de lado el aspecto de guiar el proceso de construcción de los conocimientos por parte de los mismos pequeños, apoyando su iniciativa y el deseo de experimentar, explorar y conocer por sus propios medios.

En este sentido, Hildebrand (1987) enfatiza la labor del educador como una persona que fomenta la independencia de sus estudiantes, así como la expresión de sus ideas y la espontaneidad de su curiosidad. Parece que este aspecto se está abandonando para darle prioridad a la propia acción de las docentes.

En el caso de las guarderías, en 4 grupos (66.66\%) las educadoras orientan su labor hacia la transmisión de conocimientos. De modo que sigue pendiente la necesidad de repensar las funciones de una docente, las cuales, desde una perspectiva constructivista, están enfocadas a fomentar el accionar de los párvulos y promover su desarrollo integral.

En las observaciones realizadas, se denotó que las docentes se centran en implementar rutinas de clase que les permitan trabajar con las niñas y 
los niños conceptos, temas, e incluso destrezas que ellos deben conocer y desarrollar. El trabajo docente se caracteriza por la necesidad de transmitir conocimientos, pero está carente de facilitar las experiencias dirigidas a los estudiantes para que se sean ellos quienes construyan sus propios conocimientos, con base en la experimentación, la exploración y la equivocación durante la realización de la actividad.

\section{Gráfico 2}

Estrategias metodológicas que las docentes utilizan en el aula

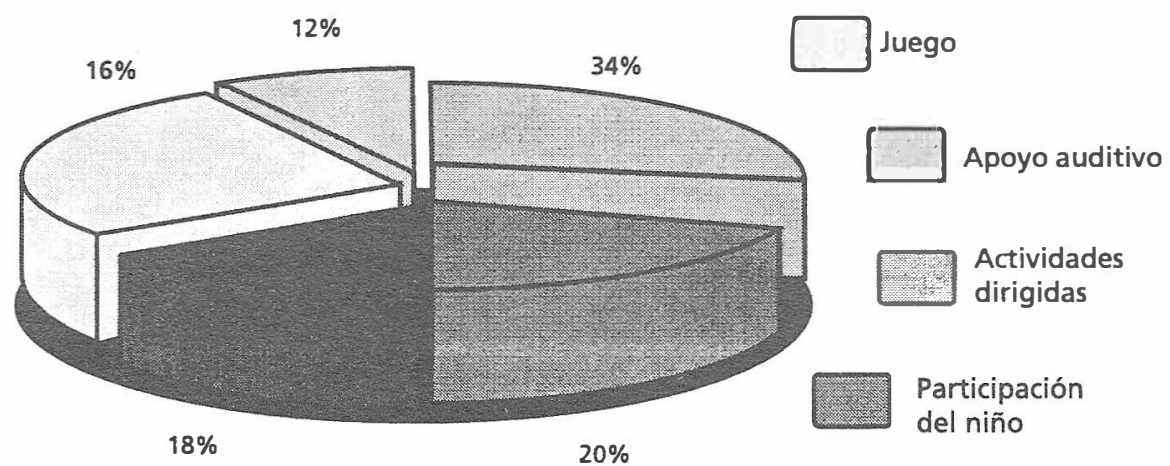

Apoyo visual

Este gráfico presenta algunas de las estrategias metodológicas, que las docentes que atienden niñas y niños de 2 a 4 años de edad, utilizan en el trabajo de aula. Es posible ubicar las respuestas en cinco categorías básicas, las cuales se distribuyen de la siguiente manera: Actividades dirigidas, Juego, Apoyo visual, Apoyo auditivo y Participación de las niñas y los niños en la dinámica del aula.

Ahora bien, el criterio denominado como actividades dirigidas es mencionado por un $34 \%$ de las docentes como la estrategia metodológica más empleada por ellas con su grupo, entre las cuales se destacan: actividades llamativas, adecuadas para los niños, de atención grupal, de acuerdo con el tema, estructuradas, activas, de concentración, dinámicas, significativas, de manejo de límites, de rutina, de estimulación constante que incluyan recompensas y premios, sensoriales y afectivas.

Las docentes manifiestan que en sus clases hay participación del niño en la rutina de clase (20\%), y entre otras actividades mencionan: la confección de material por parte de los pequeños, escogencia del tema de estudio, expresión 
de ideas por parte de sus estudiantes, exposiciones, experimentos, recetas de cocina, dramatizaciones, talleres de manualidades, meriendas compartidas, objetos de apego entre la niña o el niño y su hogar (objeto transicional: tener un objeto casero para favorecer la adaptación del niño).

El apoyo visual representa un $18 \%$ de las estrategias metodológicas mencionadas por las docentes, en las que se incluye: el uso de la computadora, láminas, material concreto, de colores llamativos, apresto, recursos educativos, material gráfico, técnicas de arte y manejo de títeres. Por otra parte, el $16 \%$ de las docentes mencionan el juego como estrategia, involucrando: el juego trabajo, semidirigido, de mesa, libre, de expresión corporal, rondas, dinámicos y enseñanza. Finalmente, un $12 \%$ de las docentes indica que como estrategia recurre al apoyo auditivo, con el uso de canciones, cambios de volumen de voz, lecturas de cuentos, consignas y rimas sencillas.

En cuanto a las interacciones, se logra detallar que en los jardines infantiles, las niñas y los niños mantienen una interacción caracterizada como buena con la docente del grupo. Esto porque en los seis grupos observados existe un contacto físico cálido, una comunicación bastante positiva y la utilización de palabras comprensibles por parte de la educadora, cuando se acerca para hablarles a sus estudiantes.

De acuerdo con las observaciones realizadas, se comprueba que existe un importante porcentaje de relaciones entre las docentes y sus estudiantes, en las que se disfruta de la compañía, las actividades y la relación entre ambos. Se logró observar que las docentes participantes en la investigación mostraron una actitud bastante positiva hacia su trabajo y el trato con las niñas y los niños, dando como resultado un tipo de relación muy especial entre estos y sus educadoras.

En las guarderías, el $50 \%$ de relaciones se catalogan como positivas, frente al $50 \%$ de relaciones en las que existe cierta interacción y comunicación con las niñas y los niños y la presencia de algunos gestos de poco disfrute del trabajo por parte de la docente. Este aspecto no se debe dejar de lado, pues se convierte en una de las luchas constantes por favorecer ambientes agradables para los párvulos y momentos de sana interacción entre estos y las docentes.

Es de suma importancia considerar que una de las necesidades de las niñas y los niños en estas edades, es la socialización. Sin embargo, para construirla, es básico encontrarse en un ambiente en el que se favorezcan relaciones positivas con las personas que los rodean. A raíz de esto, se comprende 
que en los grupos en los que se hallaron porcentajes de relaciones no del todo positivas, urge valorar la actitud de las docentes para ofrecer a los chicos la seguridad, la estabilidad y la expresión de cariño y atención, con la finalidad de mejorar las relaciones interpersonales entre educandos y educadoras.

En esta línea, se logra comprender que no sólo se trata de atender a un grupo de pequeños, sino además, de percibir sus necesidades, las características de su edad y el significado de hacerles sentir personas importantes. Por eso, y aunque en estas edades se encuentran en plena construcción de su independencia, es fundamental promover la socialización con otros párvulos y adultos de una manera agradable.

La persona que decide ser maestro asume una importante responsabilidad tanto profesional, social como emocional (León, 1998). De ahí que el modo en que efectúe su labor, influirá de manera muy significativa en la vida de sus estudiantes. Es así como, debe tomarse como un reto personal, el realizar esfuerzos cada vez más importantes en pro del bienestar de las niñas y los niños y de las mismas docentes.

\section{Consideraciones finales}

Según los resultados y la información obtenida, se concluye que la estrategia metodológica más utilizada por las docentes que atienden niños y niñas de 2 a 4 años, en su labor diaria, es aquella con actividades dirigidas. Dicho aspecto se revela con claridad en la realidad, pues la docente se encarga de establecer la rutina, las actividades, el modo de trabajo, el tiempo y el espacio.

Por el contrario, la estrategia menos usada es el juego; de hecho, no se emplea o percibe como la principal estrategia de aprendizaje para atender el proceso educativo de estos infantes. Esta situación demuestra el poco valor didáctico que se le da a la principal y más interesante actividad para las niñas y los niños. La prioridad se da en lo propuesto por la maestra.

Asimismo, la mayoría de instituciones visitadas utiliza como estrategia metodológica para la organización del aula, los espacios por áreas. Esta organización forma parte de la rutina de clase, como un período denominado juego-trabajo, metodología establecida como oficial por el Ministerio de Educación Pública, en el año 1990.

A partir de los datos obtenidos en la investigación, las docentes expresaron su opinión acerca de las características que poseen las estrategias metodológicas que utilizan cuando atienden a esta población. La mayoría de las 
respuestas caracterizaron dichas estrategias como interactivas, constructivas, aptas para la edad y concretas.

En contraste con las respuestas de las maestras, en las observaciones realizadas se encontró que la mayoría aplica estrategias monótonas, rutinarias, que no valorizan ni estimulan las potencialidades de las niñas y los niños, que no satisfagan sus intereses y necesidades, por lo que no son significativas para ellos.

Las actividades dirigidas presentaron rasgos comunes como la rutina, monotonía, con niveles de dificultad superior o inferior al de los niños que se atendían. Además, la participación de la niña o el niño fue mínima; algunas actividades eran muy estructuradas y no permitían ideas o propuestas de los y las estudiantes.

En estas actividades, se enfatizó mucho el trabajo manual, el apresto, hojas poligrafiadas y la utilización de materiales poco concretos para la edad. Varias de las experiencias se realizaron sin un objetivo específico, simplemente para entretener a los niños en el tiempo que estuvieran en la institución.

El juego se constituye en una estrategia poco empleada en el proceso educativo. Su uso se caracterizó por ser libre, principalmente, durante el recreo, también en el período de juego-trabajo; pero no se incluyeron sus valiosas cualidades como estrategia de aprendizaje. En ocasiones, esta actividad provocaba aburrimiento e insatisfacción, pues los espacios de recreo o juego-trabajo se extendían demasiado.

A pesar de que el juego esta concebido con el fin de aprovechar como una estrategia de aprendizaje, en la práctica, el juego-trabajo fue utilizado para que las y los estudiantes pasaran el tiempo, no fue un período planificado, ni con objetivos; tampoco se realizaron proyectos o tareas específicas para cumplir una meta o producto; fue sólo el hacer por hacer o jugar por jugar.

Cabe destacar que en su mayoría, las docentes encuestadas no utilizan el Programa del Ciclo Materno Infantil del Ministerio de Educación Pública; por el contrario, su mayor herramienta de trabajo es el Plan anual de la institución o, en algunos casos, guías didácticas hechas por otras personas o grupos.

No obstante, los apoyos que las educadoras utilizan, en muchas ocasiones, provocan una disociación entre lo que escriben en sus planeamientos y lo que realizan en la situación de aula. 
En relación con las definiciones que las docentes dan acerca de las estrategias metodológicas, se visualiza que tienen una idea generalizada del concepto similar a lo que la teoría define. Se puede notar que las definiciones de las docentes se relacionan con la organización y desarrollo de un tema o una lección. Sin embargo, es necesario recordar que las estrategias metodológicas orientan todos los elementos y factores del currículo.

Con respecto a los criterios que las maestras dicen utilizar para elegir las estrategias metodológicas, el principal criterio se refiere a las necesidades de sus estudiantes. Sin embargo, con las observaciones se contrapone que este es uno de los aspectos más alejados de la realidad, así como la edad y los conocimientos previos de las niñas y los niños. Como se ha mencionado, en muchos de los períodos y experiencias que se realizan en la rutina de clase, se enfatizan las decisiones tomadas por las docentes. 


\section{Referencias}

Arce, L. (2003). El uso del libro de aprestamiento para niños del Ciclo de Transición que asisten a Jardines Infantiles Públicos según la percepción de las docentes del Circuito 04 de la provincia de San José. Tesis de Licenciatura no publicada, Universidad Nacional, Heredia.

Arce, E.; Arce, F.; Brenes, M.; Camacho, E.; Meléndez, S. y Mora, N. (1999). Análisis comparativo de los Hogares Comunitarios con otras instituciones de Educación Preescolar. Seminario de Licenciatura no publicado, Universidad de Costa Rica, San José.

Bogantes, F. y Jiménez, F. (1996). Análisis de las Estrategias Metodológicas utilizadas por las maestras de aula recurso de problemas de aprendizaje (programa presupuestario 508) en la enseñanza de la lectura y la escritura. Tesis de Licenciatura no publicada, Universidad Nacional, Heredia.

Bolaños, I.; Castro, V.; Chaves, R.; Masís, Z. y Montenegro, B. (2000). Análisis de las estrategias de enseñanza que utilizan las docentes para el desarrollo de la comunicación de niños y niñas con discapacidad cognitiva los cuales tienen necesidad de apoyo limitado, integrados en el primer ciclo de la educación general básica. Tesis de Licenciatura no publicada, Universidad Nacional, Heredia.

Cerdas, A. (1995). Guía didáctica para el desarrollo del aprendizaje de niños de 0 a 3 años. San José, Costa Rica: MEP, División de Desarrollo Curricular, Dpto. de Educación Preescolar.

Cerdas, A. y Mata, A. (2000). Programa de Estudios Ciclo Materno Infantil. San José, Costa Rica: MEP.

Chaverri, L.; López, M. y Salas, M. (1993). Enfoque curricular del desarrollo socioafectivo en la Educación Preescolar: estrategias metodológicas para niños y niñas de 5 a 6 años. Seminario de Licenciatura no publicado, Universidad de Costa Rica, San José.

Chaves, G. y Villafuerte, L. (1999). El uso de los mapas conceptuales en dos jardines infantiles públicos del circuito 02 de la provincia de Alajuela: Sistematización de una experiencia. Tesis de Licenciatura no publicada, Universidad Nacional, Heredia.

Chaviano, G. y Rodríguez, A. (2003). El proyecto como estrategia de aprendizaje en el currículo preescolar con niños y niñas de 5 a 6 años de edad. Tesis de Licenciatura no publicada, Universidad Nacional, Heredia.

De Zubiría, J. y González, M. (1995). Tratado de Pedagogía Conceptual: Estrategias Metodológicas y Criterios de Evaluación. Fundación Alberto Merani. Bogotá: FONDO DE PUBLICACIONES BERNARDO HERRERA MERINO. 
Escamilla, M. y Fuentes, C. (1985). Aportes para la construcción de un modelo de capacitación para los asistentes de Educación Preescolar de los Centros Infantiles de Atención Integral. Tesis de Licenciatura no publicada, Universidad de Costa Rica, San José.

González, L. y Morales, M. (1984). Institucionalización del niño a temprana edad. Monografia de Licenciatura no publicada, Universidad Nacional, Heredia.

Hernández, R. y Rodríguez, S. (1998). Manual operativo para la evaluación y estimulación del crecimiento y desarrollo integral del niño. San José, Costa Rica: EUNED.

León, A. (1998). El maestro y los niños: la humanización del aula. San José, Costa Rica: EUNED.

Myers, R. (2000). Atención y desarrollo de la primera infancia en Latinoamérica y el Caribe: una revisión de los últimos años y una mirada hacia el futuro. Disponible en: http://www.estimulaciontemprana.com.

Obando, M. y Quesada, E. (1995). Labor pedagógica con los menores de tres años. Memoria. II Simposio de Educación Preescolar. Educar para la vida. San José, Costa Rica. Facultad de Educación. Universidad de Costa Rica.

Peralta, V. (1988). El curriculo en el Jardín Infantil: un análisis critico. Santiago, Chile: ALFA.

Salas, L. (2003). Estrategias metodológicas para promover en los niños y en las niñas de preescolar de 5 a 6 años el interés por la literatura infantil. Tesis de Licenciatura no publicada, Universidad Nacional, Heredia.

Salas, J. y Soto, E. (1996). Construyendo formas de enseñar y aprender. Heredia, Costa Rica: UNIVERSIDAD NACIONAL.

Vila, I. (2000). Aproximación a la educación infantil: caracteristicas e implicaciones educativa. Disponible en: http://www.campus-oei.org/revista/ deloslectores/468castro.pdf.

Zúñiga, I. (1995). La relación entre el conocimiento de educadores de preescolar sobre el nivel del desarrollo del niño, su preparación educativa, experiencia profesional y paternidad. Educación, 19 (2), 111-124. 